\title{
Use the Adaptive Algorithm to Estimate the Reverse Motive Force, Position and Speed of the Motor (BLDC) and Compare with the Gradient Estimation Method based on Parametric Modeling
}

\author{
Reem Toumi \\ Postgraduate student (PhD) \\ Department of Control and Automation Engineering \\ College of Electrical and Electronic Engineering
}

\author{
D. Marwan Jabra Labbad \\ Professor in University of Aleppo \\ Department of Control and Automation Engineering \\ College of Electrical and Electronic Engineering
}

\begin{abstract}
In this paper, the BLDC motor was modeled and then simulated using Simulink in the Matlab program, depending on the BLDC motor representation with state equations, and the transistor inverter that generates a three-phase motor driving signal (BLDC) was also modeled. In order to control the operation of these motors, it requires obtaining the signals of the currents of the three phases of the motor, which requires the use of three sensors (Hall), But these sensors are expensive and their use leads to noise in the current signals, so to avoid using Hall sensors, the signals of the three phase currents were guessed using the predictive algorithm, and in the next stage the BLDC motor was modeled based on parametric modeling which is characterized by obtaining an auxiliary signal. It can be used to estimate the signals of the three phase currents of the motor, and we will use the gradient method of estimating.

The adaptive algorithm for estimating the motor signals (BLDC) was compared with the gradient estimation method based on the parametric modeling when there is no load and when the motor axis is loaded with a load. And angular velocity signal, three phase current signals, and position signal.
\end{abstract}

\section{Keywords}

Motor (BLDC), Sensors (Hall), Adaptive algorithm, Gradient algorithm, Parametric modeling

\section{INTRODUCTION [1][2]}

Brushless DC motor = BLDC motors have been widely adopted in most industrial applications, with the advantage of BLDC motors compared to DC motors for long service life, high dynamic response, high efficiency, better speed and linear advantage between speed. The torque is more linear than the DC motor, in addition to the absence of acoustic noise, and the BLDC motor provides relatively more torque than the DC motor with respect to the weight change.

In order to drive and control the BLDC, an estimate of the following signals is required:

1- The reverse electromotive force signal.

2- Angular velocity signal.

3- The signals of the three phase currents.

4- Position signal.

The position of the rotor is determined sing either the Hall sensors or the use of position encoders, but the use of position sensors (Hall) has many disadvantages, the most important of which is the appearance of noise and the high price of these sensors.

In this paper, the BLDC motor was modeled in two ways, first depending on the state equations, second using the adaptive algorithm, the parametric modeling, next using the gradient estimation method, then the Simulink model simulation using the Matlab program. Finally, using the adaptive and gradual algorithms for estimating. The signals of the reverse electrical force, velocity and position, thus avoiding the use of Hall effect sensors, which are expensive and their use makes noise.

\section{THE IMPORTANCE OF PAPER AND ITS OBJECTIVES [2] [3]}

The importance of the paper is summarized in Modeling the Engine (BLDC) and its representation by state equations, as well as modeling it according to the parametric method, which is characterized by two important matters:

Modeling without using integrals that may reach saturation and in this case the modeling becomes false.

- Obtaining an auxiliary sign that can be used in estimating state variables, estimating parameters, polar displacement, designing regulators ...

- Obtaining an auxiliary sign that can be used in estimating state variables, estimating parameters, polar displacement, designing regulators ...

When estimating the reverse motive force, position and speed of the motor (BLDC) signals, and not using Hall sensors, and comparing the estimation results between the adaptive estimation method and the gradual estimation method depending on the auxiliary signal resulting from the parametric modeling.

\section{RESEARCH METHOD}

\subsection{Modeling the BLDC with State}

Equations [3]

Figure (1) shows a typical three-phase BLDC motor with its representative electrical circuit 


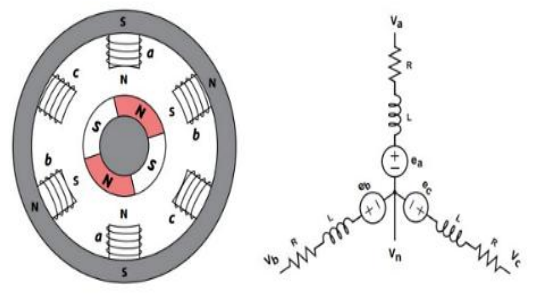

Figure (1) BLDC motor with equivalent circuit

The BLDC Mathematical Model is described by two types of electrical and mechanical equations, as follows:

Electrical equations:

$V_{a}=R \cdot i_{a}+L \cdot \frac{d i_{a}}{d t}+e m f_{a}$

$V_{b}=R \cdot i_{b}+L \cdot \frac{d i_{b}}{d t}+e m f_{b}$

$V_{c}=R \cdot i_{c}+L \cdot \frac{d i_{c}}{d t}+e m f_{c}$

Whereas:

$\left(V_{a}, V_{b}, V_{c}\right)$ The three phase voltages, $\left(i_{a}, i_{b}, i_{c}\right)$ three phase currents, $\left(e m f_{a}, e m f_{b}, e m f_{c}\right)$ the three phase reverse electromotive force.

$(R, L)$ Resistive and self-phase of the stator winding.

Where the reverse electromotive force is given by the following relationships:

$e m f_{a}=k_{e} \cdot \omega_{m} \cdot F\left(\theta_{e}\right)$

$e m f_{b}=k_{e} \cdot \omega_{m} \cdot F\left(\theta_{e}-\frac{2 \pi}{3}\right)$

$e m f_{c}=k_{e} \cdot \omega_{m} \cdot F\left(\theta_{e}+\frac{2 \pi}{3}\right)$

whereas:

$\left[k_{e}(\mathrm{~V} / \mathrm{rad} / \mathrm{sec})\right]$ Constant reverse electromotive force of the motor.

$\left[\omega_{m}(\mathrm{rad} / \mathrm{sec})\right]$ The angular velocity of the rotor.

$\left[F\left(\theta_{e}\right)\right]$ The reference function of the reverse electromotive force has a trapezoid shape with a length of amplitude $( \pm 1)$, and is given by the following formula:

$F\left(\theta_{e}\right)= \begin{cases}1 & : 0 \leq \theta_{e} \leq \frac{2 \pi}{3} \\ 1-\frac{6}{\pi}\left(\theta_{e}-\frac{2 \pi}{3}\right) & : \frac{2 \pi}{3} \leq \theta_{e} \leq \pi \\ -1 & : \pi \leq \theta_{e} \leq \frac{5 \pi}{3} \\ -1+\frac{6}{\pi}\left(\theta_{e}-\frac{5 \pi}{3}\right): & \frac{5 \pi}{3} \leq \theta_{e} \leq 2 \pi\end{cases}$

The total electromagnetic torque is given by the following relationship:

$T_{e}=\frac{1}{\omega_{m}}\left(e m f_{a} \cdot i_{a}+e m f_{b} \cdot i_{b}+e m f_{c} \cdot i_{c}\right)$
- Mechanical equation:

$T_{e}=B \cdot \omega_{m}+J \cdot \frac{d \omega_{m}}{d t}+T_{l}$

whereas:

$\left(T_{e}\right)$ Total electromagnetic torque.

(B) Friction constant torque between electrical and mechanical angle.

$(\mathrm{J}(\mathrm{V} / \mathrm{rad} / \mathrm{sec}))$ Constant reverse electromotive force.

$\left(T_{l}\right)$ The torque of the motor load.

Where the relationship between the electrical and mechanical angle is given by the following relationship:

whereas: $\left(T_{e}\right)$ Total electromagnetic torque.

(B) Friction constant torque between electrical and mechanical angle.

$(J(V / r a d / \mathrm{sec}))$ Constant reverse electromotive force.

$\left(T_{l}\right)$ The torque of the motor load.

Where the relationship between the electrical and mechanical angle is given by the following relationship:

$\left(T_{e}\right)$ Total electromagnetic torque.

(B) Friction constant torque between electrical and mechanical angle.

$(J(\mathrm{~V} / \mathrm{rad} / \mathrm{sec}))$ Constant reverse electromotive force.

$\left(T_{l}\right)$ The torque of the motor load.

Where the relationship between the electrical and mechanical angle is given by the following relationship:

$\theta_{e}=\frac{P}{2} \cdot \theta_{m}$

Where:

$\left[\theta_{m}(\mathrm{rad})\right]$ the mechanical angle of the rotor.

$\left[\theta_{e}(\mathrm{rad})\right]$ the electrical angle of the rotor.

From relationships (1) and (2) the BLDC motor mathematical modeling can be formulated with the following state equations:

$\left[\begin{array}{c}\frac{d i_{a}}{d t} \\ \frac{d i_{b}}{d t} \\ \frac{d i_{c}}{d t} \\ \frac{d \theta_{m}}{d t}\end{array}\right]=\left[\begin{array}{cccc}-\frac{R}{L} & 0 & 0 & 0 \\ 0 & -\frac{R}{L} & 0 & 0 \\ 0 & 0 & -\frac{B}{J} & 0 \\ 0 & 0 & 1 & 0\end{array}\right]\left[\begin{array}{c}i_{a} \\ i_{b} \\ i_{c} \\ \theta_{m}\end{array}\right]+\left[\begin{array}{ccc}\frac{2}{3 L} & \frac{1}{3 L} & 0 \\ -\frac{1}{3 L} & \frac{1}{3 L} & 0 \\ 0 & 0 & \frac{1}{J} \\ 0 & 0 & 0\end{array}\right]\left[\begin{array}{c}V_{a b}-e m f_{a b} \\ V_{b c}-e m f_{b c} \\ T_{e-} T_{l}\end{array}\right]$ 


$$
\left[\begin{array}{c}
i_{a} \\
i_{b} \\
i_{c} \\
\omega_{m} \\
\theta_{m}
\end{array}\right]=\left[\begin{array}{cccc}
1 & 0 & 0 & 0 \\
0 & 1 & 0 & 0 \\
-1 & -1 & 0 & 0 \\
0 & 0 & 1 & 0 \\
0 & 0 & 0 & 1
\end{array}\right]\left[\begin{array}{c}
i_{a} \\
i_{b} \\
\omega_{m} \\
\theta_{m}
\end{array}\right]
$$

Where:

$V_{a b}=V_{a}-V_{b}, \quad V_{b c}=V_{b}-V_{c}$

$e m f_{a b}=e m f_{a}-e m f_{b}, e m f_{b c}=e m f_{b}-e m f_{c}$

\subsection{Motor Driver (BLDC) - Voltage inverter [4] [5]}

The constant voltage is inverted by using the transistor inverter with the control circuit using the transistors. Figure (2) illustrates the simulation of the inverter using the (Simulink), and Table (1) shows the transistors opening pulses and the ranges of work of the transistors to obtain the threephase signals in the form of a trapezoid.

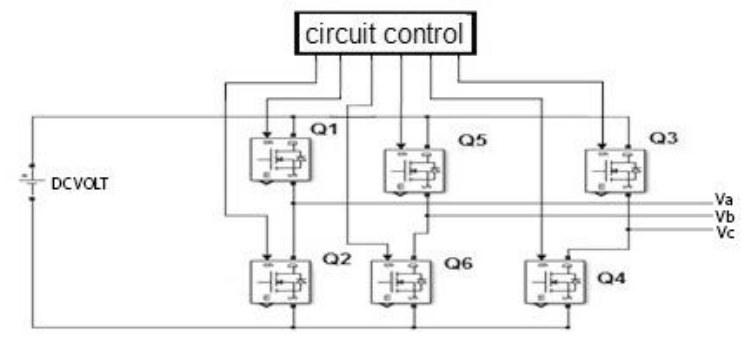

Figure (2) the transistor inverter

Table (1) Control open inverter transistors

\begin{tabular}{|c|c|c|c|c|c|}
\hline \multirow{2}{*}{ Work range } & \multicolumn{2}{|l|}{ transistors } & \multicolumn{2}{l}{ Phase currents } \\
\cline { 3 - 6 } & \multicolumn{2}{|l|}{$A$} & $B$ & $C$ \\
\hline $0^{\circ}-60^{\circ}$ & $Q_{1}$ & $Q_{4}$ & + & - & Off \\
\hline $60^{\circ}-120^{\circ}$ & $Q_{1}$ & $Q_{6}$ & + & Off & - \\
\hline $120^{\circ}-180^{\circ}$ & $Q_{3}$ & $Q_{6}$ & Off & + & - \\
\hline $180^{\circ}-240^{\circ}$ & $Q_{3}$ & $Q_{2}$ & - & + & Off \\
\hline $240^{\circ}-300^{\circ}$ & $Q_{5}$ & $Q_{2}$ & - & Off & + \\
\hline
\end{tabular}

Figure (3) shows the three-phase trapezoidal signals needed to drive the BLDC

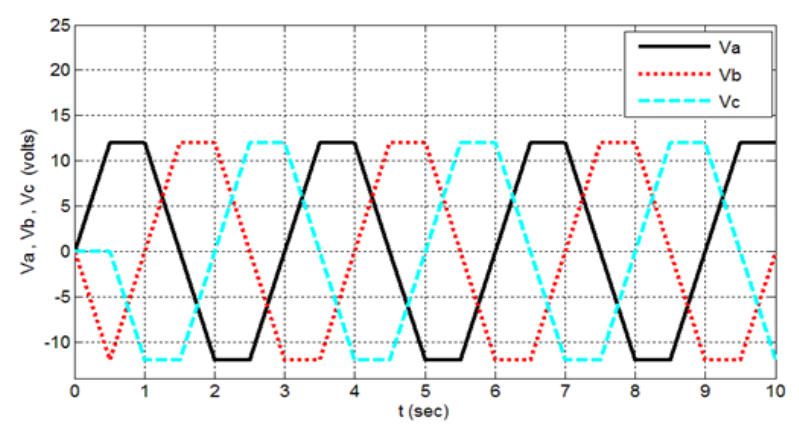

Figure (3) The three-phase trapezoidal signals needed to drive the motor (BLDC)

\subsection{Simulink Circuit of BLDC Motor with}

\section{Drive Circuit: [6] [5]}

Figure (4) shows the simulation scheme for the BLDC based on its representation by the state equations given by relationships (7) and (8), and the use of the driving circuit shown in figure (2):

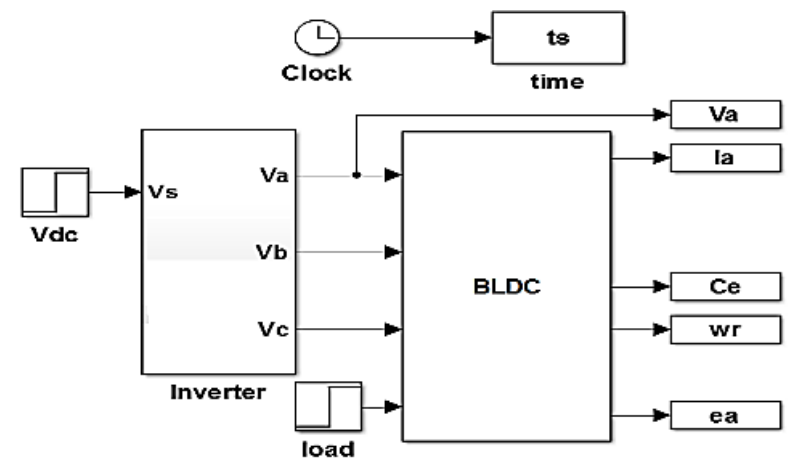

Figure (4) Simulation diagram of the BLDC

As the BLDC motor parameters are shown in Table (1)

Table (1) BLDC motor parameters

\begin{tabular}{|c|c|c|}
\hline symbol & Parameter & Value \\
\hline$K e$ & $\begin{array}{c}\text { Constant reverse } \\
\text { electromotive } \\
\text { force of the } \\
\text { motor }\end{array}$ & 0.0193 \\
\hline$R$ & $\begin{array}{c}\text { Resist the } \\
\text { armature }\end{array}$ & $0.3240 \Omega$ \\
\hline$L$ & $\begin{array}{c}\text { Self-armature } \\
2.7150 \mathrm{e}-04 \mathrm{Henrey}\end{array}$ \\
\hline$F$ & Friction factor & $4 \mathrm{e}-5 \frac{\mathrm{N} \cdot \mathrm{m} . \mathrm{sec}}{\mathrm{rad}}$ \\
\hline$J$ & $\begin{array}{c}\text { Inertia torque } \\
P\end{array}$ & $\begin{array}{c}\text { The number of } \\
\text { poles }\end{array}$ \\
\hline
\end{tabular}




\subsection{Predictive Adaptive Algorithm [6] [7] \\ [8]}

In this section, the method of estimating signals based on the laws of on-line prediction is explained, and the results are tested using the MATLAB program.

Suppose the first-degree system is given by the following differential equation:

$\dot{x}(t)+a \cdot x(t)=b \cdot u(t)$

Which can be written as follows:

$\dot{x}(t)=-a \cdot x(t)+b \cdot u(t)$

As the parameters $a, b$ are unknown, $u(t)$ the input signal, $x(t)$ the output signal or the state switch are.

Suppose the estimated parameters are $\hat{\boldsymbol{a}}, \hat{\boldsymbol{b}}$, and suppose the estimation error for the signal is:

$\varepsilon_{1}=x-\hat{x}$

Where $\hat{x}$ is the vector of the estimated state variables depending on an estimate $\hat{a}, \hat{b}$, which $\hat{x}$ is found by means of the previous equation which becomes as follows:

$\dot{\hat{x}}(t)=-\hat{a} \cdot \hat{x}(t)+\hat{b} \cdot u(t)$

The adaptive algorithm is based on finding the differential equation of error as follows:

By deriving the error equation (12), we find:

$\dot{\varepsilon}_{1}=\dot{x}-\dot{\hat{x}}$

We substitute equations (11) and (13) into equation (14) and find:

$\dot{\varepsilon}_{1}=-a \cdot x+b \cdot u-(-\hat{a} \cdot \hat{x}+\hat{b} \cdot u)$

By adding the two terms $(a \hat{x}-a \hat{x})$ to

(14), we find:

$\dot{\varepsilon}_{1}=-a \cdot \varepsilon_{1}+(\hat{a}-a) \cdot \hat{x}-(\hat{b}-b) \cdot u$

Assuming that the parameter error is defined as follows:

$\tilde{a}=\hat{a}-a, \tilde{b}=\hat{b}-b$

From it we write equation (16) in the following form:

$$
\dot{\varepsilon}_{1}=-a \cdot \varepsilon_{1}+\tilde{a} \cdot \hat{x}-\tilde{b} \cdot u
$$

In order to estimate the process represented by tracing the estimated parameters $\hat{a}, \hat{b}$ to $a, b$ to be realized, we must know the positively defined Lyapunov function, and that its derivative with respect time be negatively defined, and the Lyapunov function is chosen according to any quadratic shape, and be as follows:

$V\left(\varepsilon_{1}, \tilde{a}, \tilde{b}\right)=\frac{1}{2}\left(\varepsilon_{1}^{2}+\frac{\tilde{a}^{2}}{\gamma_{1}}+\frac{\tilde{b}^{2}}{\gamma_{2}}\right)$

And from it a derivative belonging to Lyapunov

$\dot{V}\left(\varepsilon_{1}, \tilde{a}, \tilde{b}\right)=\varepsilon_{1} \dot{\varepsilon}_{1}+\frac{\tilde{a} \dot{\tilde{a}}}{\gamma_{1}}+\frac{\tilde{b} \dot{\tilde{b}}}{\gamma_{2}}$

We substitute the secondary formula of error (18) in equation (20) and find:

$$
\dot{V}\left(\varepsilon_{1}, \tilde{a}, \tilde{b}\right)=\varepsilon_{1}\left(-a \cdot \varepsilon_{1}+\tilde{a} \cdot \hat{x}-\tilde{b} \cdot u\right)+\frac{\tilde{a} \dot{\tilde{a}}}{\gamma_{1}}+\frac{\tilde{b} \dot{\tilde{b}}}{\gamma_{2}}(21)
$$

By deriving the two relationships (17), we find:

$\dot{\vec{a}}=\dot{\hat{a}}, \dot{\tilde{b}}=\dot{\hat{b}}$

Substitute (22) into equation (21) and find:

$\dot{V}\left(\varepsilon_{1}, \tilde{a}, \tilde{b}\right)=-a \cdot \varepsilon_{1}^{2}+\tilde{a} \cdot \hat{x} \cdot \varepsilon_{1}-\tilde{b} \cdot u \cdot \varepsilon_{1}+\frac{\tilde{a} \dot{\hat{a}}}{\gamma_{1}}+\frac{\tilde{b} \dot{\hat{b}}}{\gamma_{2}}(23)$

Suppose the derivative dependent on Lyapunov according to the following formula:

$\dot{V}\left(\varepsilon_{1}, \tilde{a}, \tilde{b}\right)=-a \cdot \varepsilon_{1}^{2}$

It is negatively defined, provided that the adaptation condition $(a>0)$ is fulfilled:

$\tilde{a} \cdot \hat{x} \cdot \varepsilon_{1}-\tilde{b} \cdot u \cdot \varepsilon_{1}+\frac{\tilde{a} \dot{\hat{a}}}{\gamma_{1}}+\frac{\tilde{b} \dot{\hat{b}}}{\gamma_{2}}=0$

And from it we can impose from the relationship (25) the following:

$$
\tilde{a} \cdot \hat{x} \cdot \varepsilon_{1}+\frac{\tilde{a} \dot{\hat{a}}}{\gamma_{1}}=0,-\tilde{b} \cdot u \cdot \varepsilon_{1}+\frac{\tilde{b} \dot{\hat{b}}}{\gamma_{2}}=0
$$

Including the laws of adaptation that estimate the parameters of the control system:

$$
\dot{\hat{a}}=-\gamma_{1} \cdot \hat{\boldsymbol{x}} \cdot \varepsilon_{1}, \dot{\hat{b}}=\gamma_{2} \cdot u \cdot \varepsilon_{1}
$$

Figure (5) shows the Adaptation Law Block Diagram for estimating the parameters of the control system:

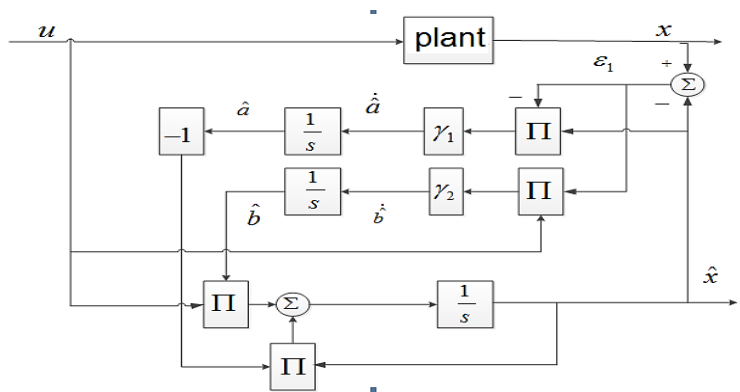

Figure (5) Block diagram for estimating parameters using an adaptive algorithm

\subsection{Application of the Predictive Algorithm to the Motor (BLDC) [9]}

By referring to equations (1), (4) and (5), we find that the estimated system is represented by the following equations

$$
\begin{aligned}
& V_{a}=\hat{R} \cdot \hat{i}_{a}+\hat{L} \cdot \frac{d \hat{i}_{a}}{d t}+e m f_{a} \\
& V_{b}=\hat{R} \cdot \hat{i}_{b}+\hat{L} \cdot \frac{d \hat{i}_{b}}{d t}+e m f_{b} \\
& V_{c}=\hat{R} \cdot \hat{i}_{c}+\hat{L} \cdot \frac{d \hat{i}_{c}}{d t}+e m f_{c}
\end{aligned}
$$




$$
\begin{aligned}
& \hat{J} \cdot \frac{d \hat{\omega}_{m}}{d t}+\hat{B} \cdot \hat{\omega}_{m}=T_{e}-T_{l} \\
& \hat{\omega}_{m}=\frac{1}{T_{e}}\left(e m f_{a} \cdot \hat{i}_{a}+e m f_{b} \cdot \hat{i}_{b}+e m f_{c} \cdot \hat{i}_{c}\right)
\end{aligned}
$$

By following the steps of the adaptive algorithm for estimating the parameters, and assuming appropriate Lyapunov functions, we obtain the adaptive laws of estimation in the following form:

$$
\begin{aligned}
& \dot{\hat{a}}=-\gamma_{1} \cdot \hat{i}_{a} \cdot \varepsilon_{1}, \\
& \dot{\hat{b}}=-\gamma_{2} \cdot\left(V_{a}-e m f_{a}\right) \cdot \varepsilon_{1}(31)
\end{aligned}
$$

Whereas, the signal error is given by the following relationship:

$$
\varepsilon_{1}=i_{a}-\hat{i}_{a}
$$

Whereas, the estimated parameters are:

$\hat{a}=\frac{\hat{R}}{\hat{L}}, \hat{b}=\frac{1}{\hat{L}}$

In the same manner, we find that the estimated parameters of equation (29) have the following formula:

$$
\dot{\hat{c}}=-\gamma_{3} \cdot \hat{\omega}_{m} \cdot \varepsilon_{2}, \dot{\hat{d}}=-\gamma_{4} \cdot\left(T_{e}-T_{l}\right) \cdot \varepsilon_{2}
$$

As the estimated parameters are:

$$
\hat{c}=\frac{\hat{B}}{\hat{J}}, \hat{d}=\frac{1}{\hat{J}}
$$

\subsection{Gradual estimation algorithm based on parametric modeling [9]}

3.6.1 Parametric Modeling [9]

Let the control system be represented by the following differential equation:

$$
\begin{aligned}
& y^{(n)}(t)+a_{n-1} y^{(n-1)}(t)+\cdots+a_{0} y(t) \\
&=b_{n-1} u^{(n-1)}(t)+\cdots \\
&+b_{0} u(t)
\end{aligned}
$$

By writing this equation in radial form, we find:

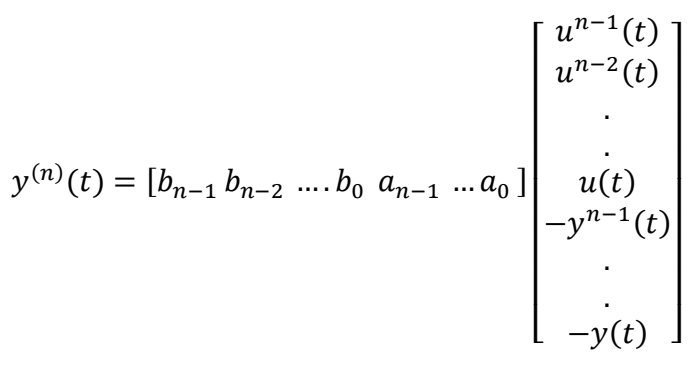$$
y^{(n)}(t)=\theta^{* T} \cdot Y(t)
$$

whereas:

$$
\begin{aligned}
& \theta^{*}=\left[\begin{array}{lllllll}
b_{n-1} & b_{n-2} & \ldots & b_{0} & a_{n-1} & \ldots & a_{0}
\end{array}\right]^{T} \\
& Y(t)=\left[u^{(n-1)}(t) u^{(n-2)}(t) \ldots u(t)-y^{(n-1)}(t) \ldots-y(t)\right]^{T}
\end{aligned}
$$

By taking the Laplace transform of the radial equation (36) we find:

$s^{n} \cdot y(s)=\theta^{* T} \cdot Y(s)$

And by taking the Laplace transform of the ray according to the relation (37), we find:

$$
Y(s)=\left[\left[s^{(n-1)} s^{(n-2)} \ldots 1\right]\left[-s^{(n-1)} \ldots-1\right] y(s)\right]^{T}
$$

Assuming the polynomial:

$\alpha_{n-1}^{T}(s)=\left[s^{(n-1)} s^{(n-2)} \ldots 1\right]$

Substitute to equation (39) we find:

$Y(s)=\left[\alpha_{n-1}^{T} u(s) \quad-\alpha_{n-1}^{T} y(s)\right]^{T}$

Substitute (41) into equation (28) and find:

$s^{(n)} y(s)=\theta^{* T}\left[\alpha_{n-1}^{T} u(s)-\alpha_{n-1}^{T} y(s)\right]^{T}$

We divide by the polynomial $\Lambda(s)$ and find

$\frac{s}{\Lambda(s)}^{(n)} y(s)=\theta^{* T}\left[\frac{\alpha_{n-1}^{T}}{\Lambda(s)} u(s) \quad-\frac{\alpha_{n-1}^{T}}{\Lambda(s)} y(s)\right]^{T}$

Where $\Lambda(s)$ is any Horvitz polynomial:

$\Lambda(s)=s^{n}+\lambda_{n-1} s^{n-1}+\cdots+\lambda_{0}$

(44)

Assuming that:

$\frac{s}{\Lambda(s)}^{(n)} y(s)=\theta^{* T}\left[\frac{\alpha_{n-1}^{T}}{\Lambda(s)} u(s) \quad-\frac{\alpha_{n-1}^{T}}{\Lambda(s)} y(s)\right]^{T}$

$z(s)=\frac{s^{n}}{\Lambda(s)} \cdot y(s)$

$\Phi(\mathrm{s})$

$=\left[\begin{array}{ll}\frac{\alpha_{n-1}^{T}}{\Lambda(s)} u(s) & \frac{\alpha_{n-1}^{T}}{\Lambda(s)} y(s)\end{array}\right]^{T}$

And from it we find after the Substitute in equation (43):

$y^{(n)}(t)=\theta^{* T} \cdot Y(t)$

It is clear that the signal Z(S) and the signal vector $\phi$ can be integrators without the need for calls by using the fractional filter:

$\frac{s^{i}}{\Lambda(s)}: i=0,1,2 \ldots, n$

Equation (44) can be written as follows:

$\Lambda(s)=s^{n}+\left[\begin{array}{lll}\lambda_{n-1} & \lambda_{n-2} & \lambda_{0}\end{array}\right] \cdot\left[\begin{array}{c}S^{n-1} \\ S^{n-2} \\ \cdot \\ \cdot \\ 1\end{array}\right]$

Assume that: $\lambda=\left[\begin{array}{lll}\lambda_{n-1} & \lambda_{n-2} & \lambda_{0}\end{array}\right]^{T}$

From proposition (40) we write the equation (47) as follows

$\Lambda(s)=s^{n}+\lambda^{T} \cdot \alpha_{n-1}(s)$

From equation (48) we find:

$s^{n}=\Lambda(s)-\lambda^{T} \cdot \alpha_{n-1}(s)$

The relationship (45) can be written after isolating the numerator parameters from the denominator parameters by the following formula:

$z(s)=\theta^{* T} \Phi(\mathrm{s})=\theta_{1}{ }^{* T} \Phi_{1}(\mathrm{~s})+\theta_{2}{ }^{* T} \Phi_{2}(\mathrm{~s})$

whereas:

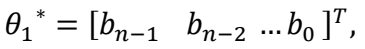


$\theta_{2}{ }^{*}=\left[\begin{array}{llll}a_{n-1} & a_{n-2} & \ldots & a_{0}\end{array}\right]^{T}$

$\Phi_{1}(\mathrm{~s})=\frac{\alpha_{n-1}^{T}}{\Lambda(s)} u(s), \Phi_{2}(\mathrm{~s})=-\frac{\alpha_{n-1}^{T}}{\Lambda(s)} y(s)$

Substitute (49) and (51) into equation (48) and find:

$y(s)=\theta_{1}^{* T} \Phi_{1}(\mathrm{~s})+\theta_{2}^{* T} \Phi_{2}(\mathrm{~s})-\lambda^{T} \Phi_{2}(\mathrm{~s})$

This relationship can be written as follows:

$y(s)=\theta_{1}^{* T} \Phi_{1}(\mathrm{~s})+\left(\theta_{2}^{* T}-\lambda^{T}\right) \Phi_{2}(\mathrm{~s})$

Figure (6) represents the parametric modeling process based on equations (50) and (53):

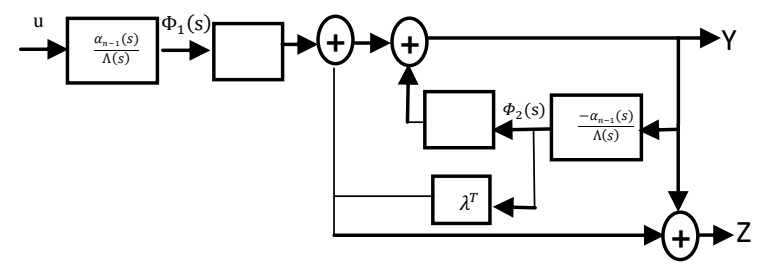

Figure (6) Block Diagram for Parametric Modeling

3.6.2 Estimating using the gradual method [9]

Parametric modeling results in the auxiliary signal that is useful in estimating state variables, designing controllers and estimating parameters, and in this paper we will use this signal to estimate the reverse motive force, position and speed of the motor, using the gradual algorithm that is given according to the following relationships:

$>$ Law of adaptation for estimating parameters: $\dot{\hat{\theta}}=\Gamma \varepsilon \phi$

$>$ Estimation error: $\varepsilon=\frac{z-\hat{z}}{m^{2}}$

$>$ Estimation auxiliary signal: $\hat{z}=\hat{\theta}^{T} . \Phi$

$>$ Design variables:

$m^{2}=1+n_{s}^{2}, n_{s}^{2}=\alpha \Phi^{T}, \alpha>0, \quad \Gamma=\Gamma^{T}>0$

\subsection{Simulink control system representation with guessing algorithm [4] [5] [6]}

By representing the state equations representing the motor (BLDC) and its driving circuit, and by representing the adaptive estimation algorithm using Lyapunov functions, as well as by representing the motor (BLDC) based on the parametric modeling and the gradual estimation algorithm representation, we obtain the Simulink diagram shown in Fig.(7):

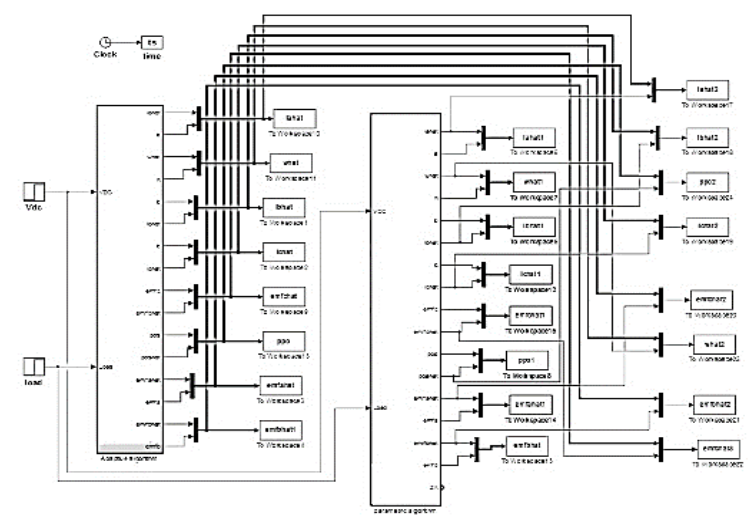

Figure (7) Simulink diagram to representing the motor (BLDC) based on the parametric modeling and the gradual estimation and the adaptive estimation

\section{SIMULATION RESULTS}

By implementing the simulink scheme shown in Figure (7), we obtain the following simulation results:

\subsection{Estimation the Reverse Electromotive Force}

Figures (8) and (9) show the estimation of the reverse electromotive force signal for the first phase of the motor (BLDC) using the adaptive algorithm and the gradual algorithm based on parametric modeling, in comparison with the true first phase reverse electromotive force, during different periods of response. As for estimating the reverse electromotive force signal for the second and third phase of the motor (BLDC), we will get signals similar to the signals shown in Figures (8) and (9), but with a shift.



Figure (8) Estimation of the first phase reverse electromotive force According to the adaptive and gradual methods

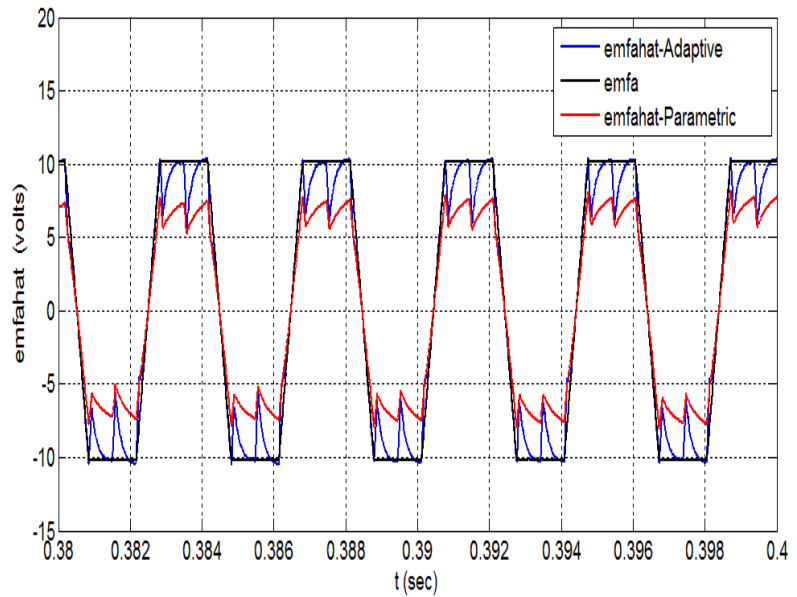

Figure (9) Estimation of the first-phase reverse electromotive force during the simulation end time (stability period) according to the adaptive and gradual methods

\subsection{Estimation the Angular Velocity}

Figure (10) shows the estimation of the indication of the angular velocity of the motor rotation axis (BLDC) according to the adaptive and gradual methods compared to the real speed. 


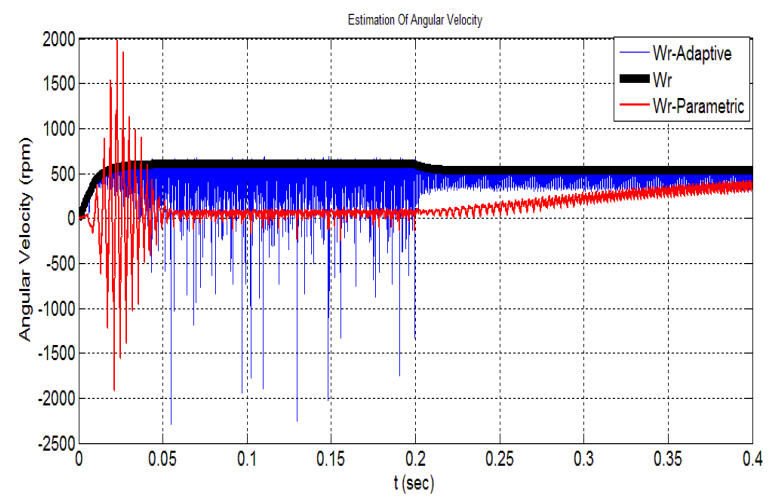

Figure (10) Estimation of the motor angular velocity (BLDC) according to the adaptive and gradual methods Compared to real speed

\subsection{Estimation the currents of the three phases}

Figures (11) and (12) show the estimation of the first phase current of the motor (BLDC) signal using the adaptive algorithm using Lyapunov functions and the gradual algorithm based on the parametric modeling, in comparison with the real first phase current, during different times of response, and for estimating the signal Currents With respect to the second and third phases of the BLDC, we will get signals similar to the signals shown in Figures (11) and (12), but with a shift.

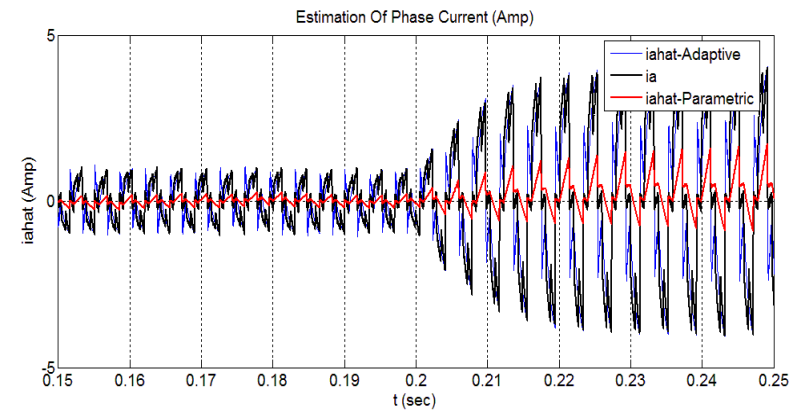

Figure (11) Estimation of the first phase current where the motor is loaded at the moment $(t=0.2 \mathrm{sec})$ according to the adaptive and gradual methods

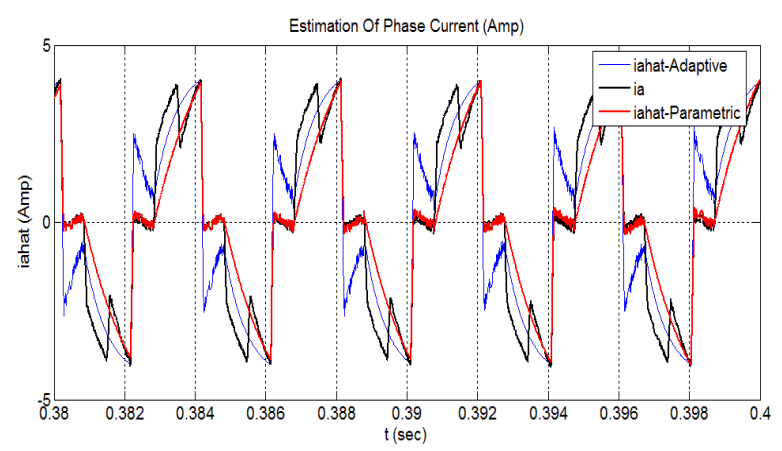

Figure (12) Estimation of the first phase current during the simulation termination time (stability period) according to the adaptive and gradual methods

\subsection{Estimation the position}

Figure (13) shows estimating the BLDC signal based on adopting a method compared to the real position, and Figure (14) shows estimating the BLDC signal according to the gradual method that depend on parametric modeling compared to the real position.

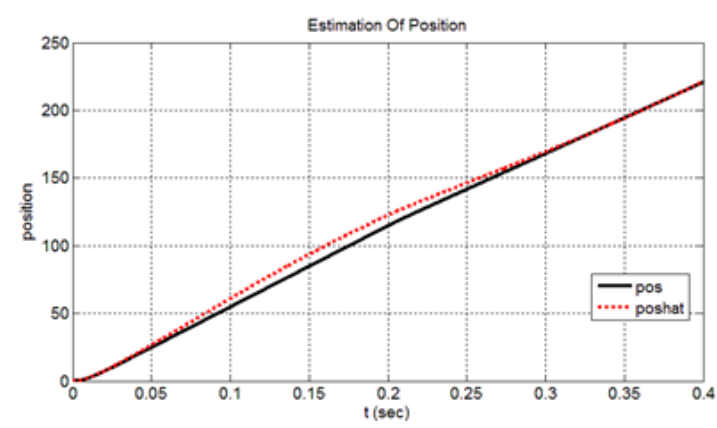

Figure (13) Estimation of the BLDC signal according to the adaptive methods Compared to the real position

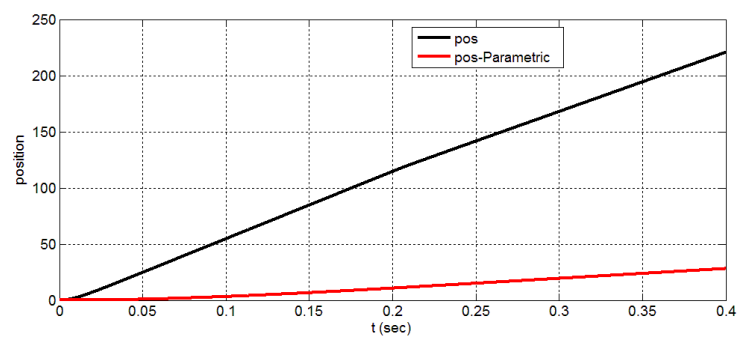

Figure (14) Estimation of the BLDC signal according to the gradual method Compared to the real position

\section{RESULTS AND DISCUSSION}

By representing the BLDC system with the adaptive algorithm and gradual algorithm, we note that both algorithms successfully estimate well the signals as shown in the previous figures, although the motor is being loaded with a load at the moment $(t=0.2 \mathrm{sec})$, the two adaptive algorithms succeeded in estimation the signals. This indicates the efficiency of these algorithms in estimating the signals despite the occurrence of external disturbances.

According to previous signals, we note the following:

$>$ The adaptive algorithm is better than the gradual algorithm, according to Figure (8), we notice that the estimation of the reverse electromotive force using the predictive algorithm is better, as the estimated signal approaches the real signal better, and after the motor is loaded, the estimation is also made better according to the adaptive algorithm.

$>$ Figure (10) illustrates estimating the speed signal, where we note the estimation by the adaptive method is better, and the estimation speed is better according to the gradual method.

From Figures (12), (13) and (14), we always note the superiority of the adaptive algorithm in the speed of estimation and its preference in comparison with the gradual algorithm.

\section{REFERENCES}

[1] Ameer L.Saleh University of Misan.And Adel Obed, Middle Technical University, "Speed Control of Brushless DC Motor based on Fractional Order PID Controller", Research• June 2014.

[2] Santanu Mondal T echno India Saltlake,And Arunabha Mitra Heritage Institute of Technology,And Madhurima Chattopadhyay Heritage Institute of T echnology," Mathematical modeling and simulation of 'Brushless DC 
motor with ideal Back EMF +for a precision speed control", Conference Paper• August 2015.

[3] Sudhanshu Mitra, R.Saida Nayak, Ravi Prakash," Modeling and Simulation of BLDC Motor using MATLAB/SIMULINK Environment",Volume: 02 Issue: 08 | Nov-2015

[4] Miss Avanti B.Tayade-Department of Electrical Engineering, ,S.D.College of Engineering \& Technology.,Wardha," Modeling and Simulation of A Bldc Motor By Using Matlab/Simulation Tool",EERA2014.

[5] Yogini B. Hirave, Prof. R.T. Patil, Mrs Ketan Bagade-
"Brushless DC Motor speed controller using DSP Processor approach",www.ijesi.org Volume 2 Issue 6 || June. 2013.

[6] Ogata K, "Modern Control Engineering", Pearson Education International, 2002.

[7] Kuo B.C. and Golnaraghi F., "Automatic Control Systems", Eighth Edition, John Wiley \& Sons, 2003.KokotoviđIc, P.V. (Ed.), "Foundations of Adaptive Control", Springer-Verlag, New York, 1991.

[8] Dr.Olaby Usama "Adaptive control engineering", university of Aleppo. 